\title{
Improving Performance of a Space-Time Turbo Code in a Rayleigh Fading Channel
}

\author{
Everest Huang, Alan Gatherer, Tarik Muharemovic, Dale Hocevar \\ Wireless Communications Branch DSPS R \& D Center \\ Texas Instruments, Inc., Dallas, TX 75243 \\ [huang, gatherer, tarik, hocevar] @ti.com
}

\begin{abstract}
Starting with an ordinary parallel concatenated turbo encoder in a block fading Rayleigh channel, we introduce bit-interleaving of the outputs, not for time diversity, but to increase the coding gain. We develop a guideline metric for designing QPSK space-time codes which reveals both the coding and diversity advantage of the system, as well as shows that it is unnecessary to guarantee full space diversity to achieve good performance in Rayleigh fading. Using channel a priori information in the MAP iterations for decoding, we achieve frame error rates within $1 \mathrm{~dB}$ of the outage probability with only modest increases in decoding complexity.
\end{abstract}

\section{INTRODUCTION}

In the harsh environment of a wireless channel, deep fades caused by multipath interference can severely degrade performance. Transmit diversity is an effective way to improve error rates, especially when having multiple receive antennas would be difficult (e.g., when transmitting from a base station to a mobile phone).

Turbo codes [1] are a class of codes that are able to achieve error rates very close to capacity, but they typically require very large block sizes, which can be undesirable in delay sensitive applications such as voice or video. Orthogonal space-time codes described in [2] only require block lengths as long as the number of antennas, but do not exist for arbitrary numbers of transmit antennas. Furthermore, [3] and [4] show that spacetime turbo codes are able to achieve superior performance to space-time codes for the same block lengths in both the timevarying and static block fading scenarios.

Starting with the system in [4], we first create our own simulation and compare results to check for accuracy. Then, after establishing a baseline curve, we add several improvements based on our analysis to improve the frame error rate (FER) of the system in a block fading Rayleigh environment.

A guideline diversity metric is developed to help improve coding performance, leading to channel interleavers being added even in the static fading case. Additionally, channel $a$ priori probability calculations are introduced into the turbo decoder to significantly increase performance. The effects of bit versus symbol level granularity in the extrinsic and channel $a$ priori calculations are explored, as well as how the QPSK mapping and the order of decoding affect the performance of the code.

For space-time turbo coded systems, several metrics have been devised (e.g. [4], [5]) to ensure that the outputs of the turbo coder are guaranteed to have full diversity. We do not, however, make any attempts to guarantee full diversity, and instead argue that good performance can still be achieved because the probability of losing diversity for a given code word be-

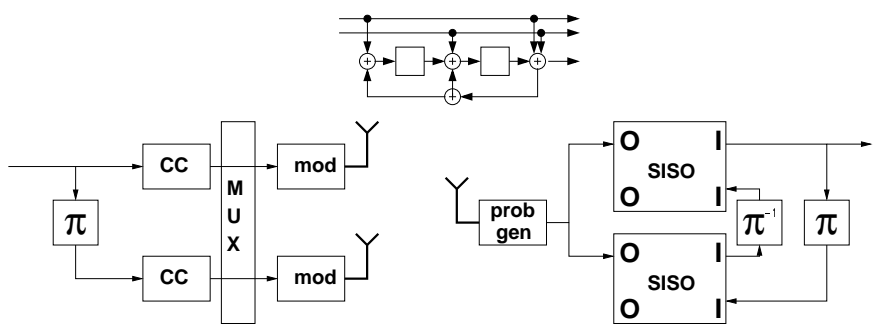

Fig. 1. The baseline system with the rate $2 / 3$ convolutional code used in the CC blocks.

comes very small even for modest block sizes. For random-like codes, it is our experience that diversity is lost rarely enough that it makes no significant impact on performance.

\section{BASELINE SYSTEM}

The system in Fig. 1 uses two rate 1/2 covolutional coders in a parallel concatenated convolutional coder (PCCC) configuration, which resembles a common turbo code. The rate $2 / 3$ convolutional code used is also shown in Fig. 1, with the first systematic bit punctured in the upper code, and the second systematic bit punctured in the lower code. The outputs of each coder are then fed through a channel interleaver to spread out the bits over several fades assuming a time varying channel and then mapped as QPSK symbols to their respective transmit antennas. There is also an optional multiplexer which alternately directs the output symbols of a code to its own antenna or to the antenna on the other branch. The reasoning for this multiplexer is allow the outputs of each convolutional coder to experience the fades of both transmit antennas. As [4] show, however, this multiplexing actually decreases performance by allowing a deeply faded antenna to cause errors in both branches. In order to calibrate the performance of our system, however, we use the same unoptimized version of the encoder.

Fig. 2 compares our results with those published in [4] for a static block fading Rayleigh channel with a blocksize of 260 information bits and a random interleaver. No channel interleaver is used, and the outputs of the convolutional coders are multiplexed. We show good agreement with their results, and then remove the multiplexing to create our baseline FER curve.

\section{Guideline Diversity Metric}

In order to design effective changes to the turbo space-time coder, we first want to develop a metric to help us decide if a code will perform well or not. Let's assume that we have two transmit antennas, both using identical QPSK constellations. 


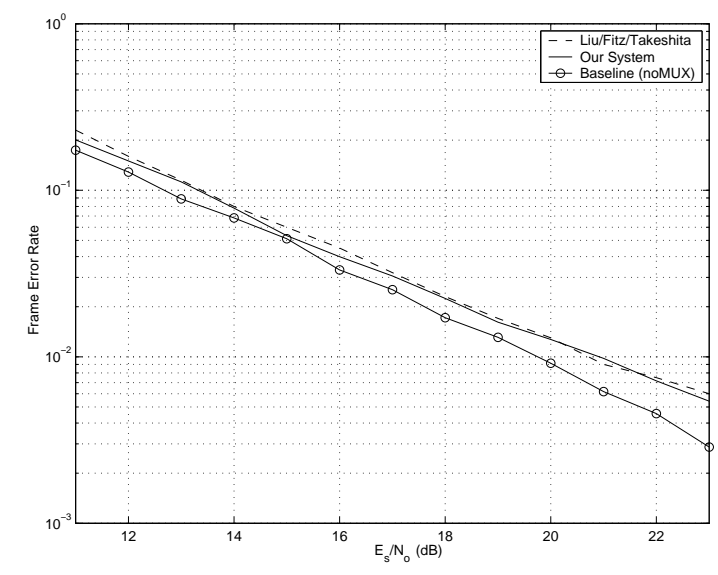

Fig. 2. Simulation comparison with published system, as well as the baseline curve.

We know from [2] that for full diversity code words, the probability of mistaking $C$ for $\tilde{C}$ is

$$
\operatorname{Pr}(C \rightarrow \tilde{C})<\left(\frac{E_{s}}{N_{0}}\right)^{-2} \frac{1}{\left|F^{*} F\right|},
$$

where $E_{s} / N_{0}$ is the signal to noise ratio (SNR), the exponent shows the diversity advantage, $F$ is the Euclidean difference between the two code words $C$ and $\tilde{C}$, and $\left|F^{*} F\right|$, is the coding advantage. Full diversity is lost when $F$ does not have rank 2, but for useful blocksizes, the probability of this is very low, as our own experience shows. We will therefore assume full diversity, and try to increase the coding advantage.

Assuming the usual mapping from a bit pair (dibit) $c$, to a QPSK symbol $C(00 \rightarrow 1+j, 01 \rightarrow 1-j$, etc $)$, we calculate $f$, the difference between two code words $c$ and $\tilde{c}$ in binary space and $F$, the difference between two code words $C$ and $\tilde{C}$ in Euclidean space. These are summarized in Table I. We see

TABLE I

SUMMARY OF $f$ AND $F$ PROPERTIES.

\begin{tabular}{c|c||c|r}
$\mathrm{c}$ & $\mathrm{C}$ & $\mathrm{f}=\mathrm{c} \oplus \tilde{\mathrm{c}}$ & $\mathrm{F}=\mathrm{C}-\tilde{\mathrm{C}}$ \\
\hline 00 & $1+j$ & 00 & 0 \\
01 & $1-j$ & 01 & $\pm 2 j$ \\
10 & $-1+j$ & 10 & \pm 2 \\
11 & $-1-j$ & 11 & $\pm 2 \pm 2 j$
\end{tabular}

that $|F|^{2}=4 d_{H}(f)$ where $d_{H}(f)$ is the Hamming weight of $f$. If we perform this operation on each dibit $F_{1}$ and $F_{2}$ from the upper and lower antennas, we can identify 4 possibilities:

$$
\begin{array}{rll}
d_{H}\left(f_{1}\right)=0 \text { or } d_{H}\left(f_{2}\right)=0 & \Rightarrow & F_{1}^{*} F_{2}=0 \\
d_{H}\left(f_{1}\right)=d_{H}\left(f_{2}\right)=1 & \Rightarrow & F_{1}^{*} F_{2}= \pm 4 j \\
d_{H}\left(f_{1}\right)+d_{H}\left(f_{2}\right)=3 & \Rightarrow & F_{1}^{*} F_{2}= \pm 4 \pm 4 j \\
d_{H}\left(f_{1}\right)=d_{H}\left(f_{2}\right)=2 & \Rightarrow & F_{1}^{*} F_{2}= \pm 8 \pm 8 j .
\end{array}
$$

From these cases, we see that $\left|F_{1}^{*} F_{2}\right|=4 \sqrt{d_{H}\left(f_{1}\right) d_{H}\left(f_{2}\right)}$.

We define $d_{1}$ to be the total Hamming weight of the upper antenna, and $d_{1, i}$ to be the Hamming weight of the $i$ th symbol of the upper transmit antenna,

$$
\begin{aligned}
\left|F^{*} F\right| & =\left\|F_{1}\right\|^{2}\left\|F_{2}\right\|^{2}-\left\|F_{1}^{*} F_{2}\right\|^{2} \\
& =\left(\sum_{i}\left|F_{1, i}\right|^{2}\right)\left(\sum_{i}\left|F_{2, i}\right|^{2}\right)-\left\|\sum_{i} F_{1, i}^{*} F_{2, i}\right\|^{2} \\
& \geq 16\left(\sum_{i} d_{1, i}\right)\left(\sum_{i} d_{2, i}\right)-\left\|\sum_{i}\left|F_{1, i}^{*} F_{2, i}\right|\right\|^{2} \\
& =16\left[d_{1} d_{2}-\left\|\sum_{i} \sqrt{d_{1, i} d_{2, i}}\right\|^{2}\right] .
\end{aligned}
$$

Thus, the error probability is inversely proportional to (3), decreases if $d_{1} d_{2}$ grows larger, and increases if the right hand sum increases. Unlike in the BPSK case of [6], this degradation is not simply dependant on the number of places that the Hamming weight of the upper and lower antennas match. We can, however, note that for a given symbol, the degradation is essentially monotonic with the weight, which suggests two design rules.

First, for a given code word, we should try to equalize $d_{1}$ and $d_{2}$ as much as possible. Second, we should minimize the weight for each dibit symbol to ensure that the bits are not too "clumpy" in a given code word (i.e., the ones should not be unevenly spread throughout the code word). This is certainly not the optimal strategy, but it does provide a guideline in the case of large code words which make exhaustive searches impossible.

In order to try to keep the symbol weights small, we use random channel interleavers to scramble the outputs of the coders [6]. Thus, the channel interleavers should improve the performance of the system even if the channel is constant over the entire block length. Equalizing $d_{1}$ and $d_{2}$ is difficult given random input, but the interleavers make the encoder outputs fundamentally different, so the position of ones are not well correlated between the two branches.

\section{Using Channel A Prioris}

The soft-input, soft-output (SISO) modules in the turbo decoder take in a priori probabilities of the input bits and the output symbols of the convolutional code, and outputs a posteriori probabilities by using the MAP algorithm [7]. For a PCCC, the a priori probabilities of the output symbols are calculated from the received values from the channel.

In an ordinary turbo decoding procedure for a block of data, the output a priori probabilities are the probability that a given constellation point was sent given the received symbol. These are fed to each SISO module, and remain constant for all of the iterations. The iterative improvment of the estimates of the input bits are thus only caused by the cross-coupling of the SISO modules' input a posteriori outputs, therefore updating the out- 

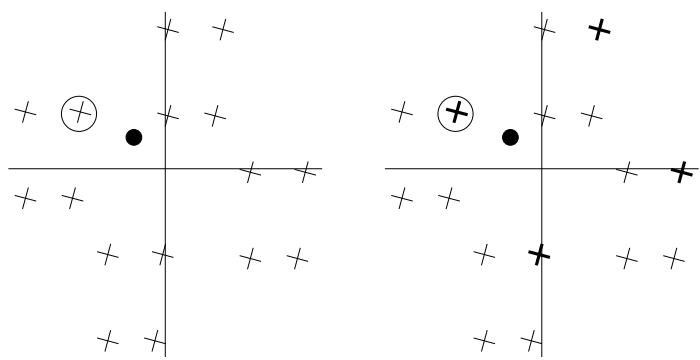

Fig. 3. Sample 2x1 QPSK constellation (left), and with channel a priori information (right). The circled constellation point is the actual transmission, and the solid circle is the received noisy signal.

put a priori probabilities on each iteration should improve performance.

If there are two transmit antennas using QPSK to transmit across a flat-fading Rayleigh channel (and assuming the antennas experience independent fades), the resulting symbol at the receive antenna will be a point in a random sixteen-point constellation in the absence of noise, as shown in the left side of Fig. 3. The circled constellation point is the actual transmission, and the solid circle is the received signal. The noise has pushed the received signal far enough away from the transmitted point that it is now closer to a different constellation point.

The probability generator that feeds the SISO output a prioris normally calculates the probability that the received symbol $z$ was produced by the transmit antennas sending $x_{1}$ and $x_{2}$, or $P\left(z \mid x_{1} x_{2}=c_{i} c_{j}\right)$ where $c_{i}$ and $c_{j}$ are points in the constellation $\mathcal{C}$. By Bayes' rule,

$$
\begin{aligned}
P\left(z \mid x_{1} x_{2}=c_{i} c_{j}\right) & =\frac{P\left(x_{1} x_{2}=c_{i} c_{j} \mid z\right) P(z)}{P\left(x_{1} x_{2}=c_{i} c_{j}\right)} \\
& \propto P\left(x_{1} x_{2}=c_{i} c_{j} \mid z\right) .
\end{aligned}
$$

Assuming equiprobable constellation points, and given that $P(z)$ is present in the equation for all constellation points, we see that $P\left(z \mid x_{1} x_{2}=c_{i} c_{j}\right)$ and $P\left(x_{1} x_{2}=c_{i} c_{j} \mid z\right)$ differ only by a constant. The probability generator then calculates output a prioris by

$$
P\left(z \mid x_{1}=c_{i}\right)=\sum_{c_{j} \in \mathcal{C}} P\left(x_{1} x_{2}=c_{i} c_{j} \mid z\right),
$$

and similarly for $P\left(z \mid x_{2}=c_{j}\right)$. Assuming the upper SISO is run first, the execution path for the probabilities is probgen $\rightarrow$ $\mathrm{SISO} 1 \rightarrow \mathrm{SISO} 2 \rightarrow$ SISO 1 , etc.

This algorithm does not use any of the updated information about the encoder outputs produced by the MAP, which are usually discarded or not calculated. Instead of using (4), we can manipulate the probabilities to allow the updated probabilities to be used. Starting again from $P\left(z \mid x_{1} x_{2}=c_{i} c_{j}\right)$, we can sum across all possible values of $x_{2}$ and assuming the antenna symbols are independent,

$$
P\left(z \mid x_{1}=c_{i}\right)=\sum_{c_{j} \in \mathcal{C}} P\left(z, x_{2}=c_{j} \mid x_{1}=c_{i}\right)
$$

$$
=\sum_{c_{j} \in \mathcal{C}} P\left(z \mid x_{1} x_{2}=c_{i} c_{j}\right) P_{a}\left(x_{2}=c_{j}\right) .
$$

Using Bayes' rule, we get

$$
\begin{aligned}
P\left(z \mid x_{1}=c_{i}\right) & =\frac{P\left(z \mid x_{1}=c_{i}\right) P_{a}\left(x_{1}=c_{i}\right)}{P(z)} \\
& \propto P\left(z \mid x_{1}=c_{i}\right) P_{a}\left(x_{1}=c_{i}\right),
\end{aligned}
$$

where we again recognize that $P(z)$ is in every equation. Note that the $P_{a}\left(x_{1}=c_{i}\right)$ and $P_{a}\left(x_{2}=c_{j}\right)$ terms are the $a$ posteriori probabilities that were output by the SISO modules during the previous iteration. An identical analysis can be made for the other transmit antenna with the roles of $x_{1}$ and $x_{2}$ reversed.

Using the a posteriori probabilities from the SISO decoders can be likened to interference cancellation of the other antenna. The upper antenna sends a QPSK constellation, but the QPSK output of the lower antenna spreads each constellation point from the upper antenna into four distinct points, effectively interfering with the uper antenna. The received signal will therefore be one of a randomly generated sixteen point constellation.

The a posteriori probabilities from the SISO decoder for the second antenna will accentuate points in the random constellation based on the probabilities that it calculates, as shown in the right side of Fig. 3. Without using the channel a prioris (the left side of Fig. 3), the maximum-likelihood (minimum-distance) detector would decide on the wrong constellation point.

On the right, since the lower SISO module has calculated which symbol the second antenna was mostly likely to have sent, the four points associated with the second antenna sending that symbol are emphasized. The a prioris have in effect cancelled the output of the second antenna. The maximumlikelihood decoder, if constrained to those four points, would choose the correct symbol for the upper antenna. Of course, in actuality that kind of hard decision would degrade performance. By using the output a posterioris, we are exploiting the fact that after the first iteration, the probabilities of the constellation points are no longer equal. Again assuming that the upper SISO is run first, the execution path for the probabilities is probgen $\rightarrow$ SISO1 $\rightarrow$ SISO2 $\rightarrow$ probgen $\rightarrow$ SISO1, etc.

Thus, by modestly increasing the complexity of the probability generator module and adding some extra data paths, we are able to use all of the information generated by the SISO modules. This method works even if the constellations of the transmit antennas are different sizes, and can easily be extended to more than two antennas. These operations scale linearly with the size of the output constellations and the number of transmit antennas, and since the processing only depends on a single symbol at a time, it also scales linearly with the block size.

\section{Other Performance Enhancements}

In addition to adding the channel interleavers and using channel aprioris, there were also several small performace tweaks that were implemented and tested. 


\section{A. Bit vs. Symbol Extrinsics and A Prioris}

Since we know that the inputs to the encoders are uncorrelated bits, it would be better to calculate input extrinsics at the bit-level rather than at the symbol level. The outputs of the convolutional coders are symbols, so it appears it would be better to calculate output extrinsics at the symbol level. However, given that outputs of the coders are punctured, the symbols are already altered at the bit level, so it turns out that bit-level output extrinsics also should improve performance.

For the a priori probabilities fed to the channel probability generator, one could also envision extending the interference cancellation idea to the actual bits of each coder's output symbol, effectively using the other three bits of the symbol to help decide on which of the two remaining constellation points is more likely. This is misleading, however, because the QPSK symbols transmitted by the antennas experience different fades, but the bits in each symbol do not. Additionally, bit-level calculations here would destroy any joint information about the two bits in each symbol, therefore symbol-level calculations are the better choice.

\section{B. QPSK Mapping}

Given the results of [8], we might find that different mappings of the bits to QPSK symbols would affect performance. For QPSK, there are only 3 distinct mappings of bits into symbols, all others being rotations. Gray coding is 0231 going around the circle, "natural" coding is 0123, and "reversed" coding is 0213 . Gray coding turns out to have the best performance, which is not surprising, given that the convolutional coder used is optimized for linear codes [9]. This does not rule out, however, that a different coder jointly optimized with the mapping (especially for higher order constellations) might give better overall performance.

\section{Ordered Decoding}

When there are only two constituent codes in the PCCC, there are only two choices on the order to run the SISO modules. Once one SISO is chosen, they alternate until some stopping criterion is reached. The usual choice in decoding is to start arbitrarily. In a gaussian channel this is appropriate, since the output of each coder experiences the same channel. In a fading environment, however, the order of decoding makes a difference. By classifying the antennas in order of strength according to the magnitude of the fading coefficient experienced by each antenna, we could either choose to decode the stronger antenna first, or the weaker, or we could just arbitrarily choose one regardless of the channel conditions, as in the AWGN case. With more than two SISO modules, then we could decide at the beginning of each iteration the optimal ordering of the decoders.

\section{Simulation Results AND Discussion}

The improved encoder/decoder is shown in Fig. 4. The addition of the channel interleavers and the feedback paths the
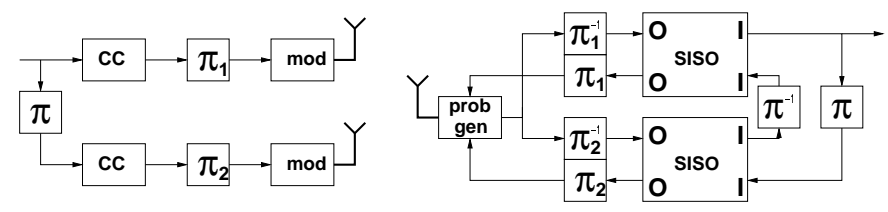

Fig. 4. The improved system, with channel interleavers and feedback paths.

the channel probability generator are the most notable changes. Because [4] shows that multiplexing the encoder outputs actually decrease performance, and from our own experience, we use separate interleavers for each encoder, to keep from "crossing the streams." The interleavers were all created using the random number generator of MATLAB, and the systems were simulated using 500 errors or $50 \times 10^{6}$ input bits as a stopping criterion. Each block experiences i.i.d. fades on each antenna which are constant over the length of the block, and the decoders were run for 10 iterations.

Fig. 5 shows the effects of using bit vs. symbol extrinsics in the baseline system of Fig. 2. The curves represent the FER after each iteration of the decoder. We can see that with just this simple change in the SISO module, we are able to gain almost $1 \mathrm{~dB}$ in FER.

In Fig. 6 we show the effect of decoding order on bit error rate (BER). Of the three choices, starting the decoding with the "weaker" antenna (with the fading coefficient that is smaller in magnitude) gives the best performance. The curves in Fig. 6 compare the arbitrary decoding (in this case, always decoding the upper antenna first) with decoding the weaker antenna first. We see about a $0.5 \mathrm{~dB}$ gain in BER by decoding the weaker antenna first. This is also a very simple modification to the decoding algorithm, and it does manage to squeeze out a little more performance for almost no effort.

Because the first SISO that is run has as its input a prioris equal probabilities for all input symbols, it would seem like decoding the stronger antenna first would be beneficial because it would be more likely to make correct estimates of the probabil-

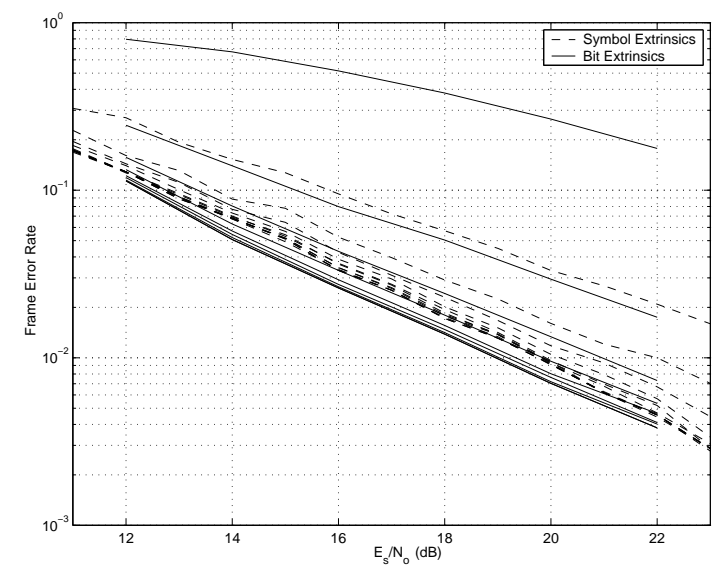

Fig. 5. Symbol extrinsics vs. bit extrinsics 


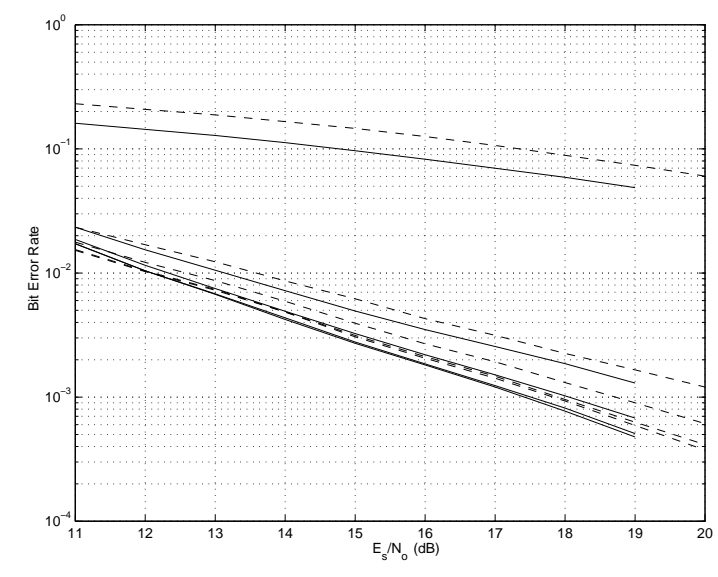

Fig. 6. Using the "weaker" (solid line) decoder first compared to choosing arbitrarily (dashed line).

ities given only the output a prioris. The second SISO and both SISOs in all subsequent iterations get as input $a$ prioris the $a$ posteriori probabilities generated by the other SISO.

If both antennas experience closely matched fades in amplitude, this may give the stronger antenna too much of a head start, and it becomes more difficult for the weaker antenna to affect the probabilities of the stronger one. This defeats the purpose of the second encoder, which helps to correct errors in the first encoder. If the weaker antenna is decoded first, then in some sense it has a half iteration head start in the decoding process. This puts the two SISO modules on more level ground, allowing each to better help the other correct errors. If the stronger antenna is much stronger, then the weaker antenna would not have much effect anyway, so the ordering would not be important in these cases.

Fig. 7 is a comparison of the effects of using the channel $a$ prioris as well as the channel interleavers on the FER of the system. The results in this figure were all with systems that always decoded the upper antenna first, due to time constraints and the small effect of the ordering in this SNR range. The outage probability is approximate in this figure, and taken from the graph in [4]. The dashed line is the baseline curve we calculated in Fig. 2, and the line with circles is the same baseline calculated using bit extrinsics instead of symbol extrinsics as with the dashed line. It is apparent that using bit extrinsics is superior to using symbol extrinsics in every case, and that using bit a prioris suffers slightly compared to using symbol $a$ prioris. Thus the best results are produced when using bit extrinsics and symbol a prioris with channel interleaving, resulting in a FER within $1 \mathrm{~dB}$ of the outage probability.

Note also that the slope is very close to -2 , which would be the full diversity slope, but we have not guaranteed diversity in any way. Because the blocksize and the channel interleavers make it very improbable that the two antennas would transmit symbols that cause a loss of diversity, we can get the same kind of performance as if we had worked to gain full diversity. This allows us to optimize the interleaver for other performance

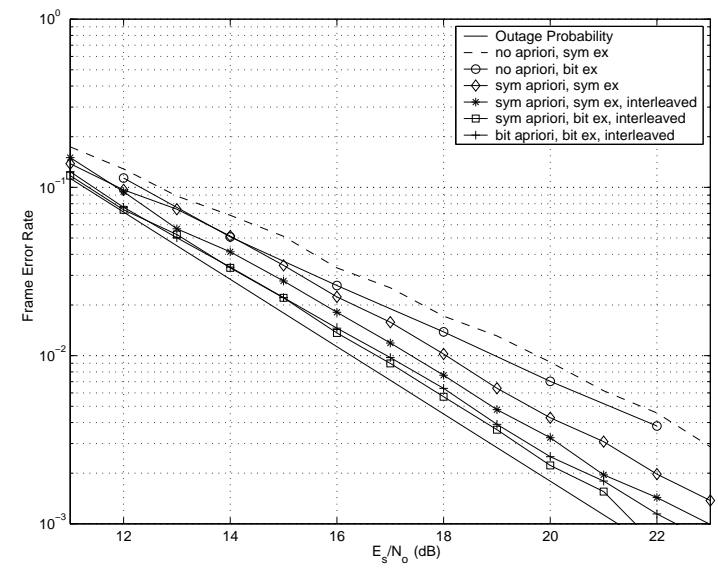

Fig. 7. Comparison of several of the techniques used.

gains without restrictions.

\section{CONCLUSIONS}

By using channel a prioris and using the guideline metric we developed, we were able to take an existing turbo spacetime system and significantly improve performance with only modest gains in complexity. We also show that it is not necessary to guarantee diversity in the encoder. The techniques used here are applicable to any other wireless system.

\section{ACKNOWLEDGMENTS}

The authors would like to thank Y. Liu for generously sharing with us their random interleaver for testing purposes.

\section{REFERENCES}

[1] C. Berrou, A. Glavieux, and P. Thitimajshima, "Near shannon limit errorcorrecting coding and decoding: Turbo-codes," in Proc. IEEE International Conference on Communication (ICC), Geneva, Switzerland, May 1993, pp. 1064-1070.

[2] V. Tarokh, N. Seshadri, and A. R. Calderbank, "Space-time codes for high data rate wireless communication: performance criterion and code construction," IEEE Trans. Inform. Theory, vol. 44, no. 2, pp. 744-765, Mar. 1998.

[3] A. Stefanov and T. M. Duman, "Turbo coded modulation for wireless communications with antenna diversity," in Proc. IEEE Vehicular Technology Conference (VTC), Amsterdam, The Netherlands, Sept. 1999, pp. 1565-1569.

[4] Y. Liu, M. P. Fitz, and O. Y. Takeshita, "QPSK space-time turbo codes," in Proc. IEEE International Conference on Communications (ICC), New Orleans, LA, June 2000, pp. 292-296.

[5] A. R. Hammond and J El Gamal, "On the theory of space-time codes for PSK modulation," IEEE Trans. Inform. Theory, vol. 46, no. 2, pp. 524542, Mar. 2000

[6] T. Muharemovic, A. Gatherer, W. Ebel, S. Srihosour, D. Hocevar, and E. Huang, "Bit-interleaved space-time codes," unpublished.

[7] S. Benedetto, D. Divsalar, G. Montorsi, and F. Pollara, "A soft-input softoutput app module for iterative decoding of concatenated codes," IEEE Commun. Lett., vol. 1, no. 1, pp. 22-24, Jan. 1997.

[8] E. N. Onggosanusi, A. Gatherer, and A. G. Dabak, "Turbo trellis-coded modulation with time varying mixed mapping," in Proc. IEEE Vehicular Technology Conference (VTC), in press.

[9] S. Benedetto, R. Garello, and G Montorsi, "A search for good convolutional codes to be used in the construction of turbo codes," IEEE Trans. Commun., vol. 46, no. 9, pp. 1101-1105, Sept. 1998. 